\title{
The Indus3Es Project: New technologies for Utilization of Heat Recovery in Large Industrial Systems
}

\author{
Patrizia Circelli $^{1}$, Valentina Cinti ${ }^{2}$, Laura Alonso $^{3}$, Asier Martinez ${ }^{3}$ \\ ${ }^{1}$ CiaoTech PNO \\ Via Colajanni 4, Rome, Italy \\ P.Circelli@ ciaotech.com \\ ${ }^{2}$ CiaoTech PNO \\ Via Pacini 11, Milan, Italy \\ V.Cinti@ciaotech.com \\ ${ }^{3}$ Tecnalia Research and Innovation \\ Area Anardi 5, 20730 Azpeitia, Guipuzcoa, Spain \\ laura.alonso@tecnalia.com, asier.martinez@tecnalia.com
}

\begin{abstract}
The Indus3Es project received funding under H2020-EE-18-2015: New technologies for utilization of heat recovery in large industrial systems, considering the whole energy cycle from heat production to transformation, delivery and end use. Funded under the Grant Agreement 680738, the main objective of the project is to develop an economically viable solution for industry, appropriate for existing plants and adaptable to various industrial processes, consisting on the technology of Absorption Heat Transformer. The developed system will be demonstrated in real environment in Tüpras, the main petrochemical industry in Turkey, enabling to analyze besides integration aspects, operational and business issues. Indus3Es System will be defined and optimized for different specificities in different sectors and industrial processes, for which up-scaling of the demonstrated technology and replication studies will be performed.
\end{abstract}

Keywords: Absorption Heat Transformer (AHT), Waste heat recovery, Industrial Process, Prototype

\section{Introduction}

Indus3Es system consists on the development of an Absorption Heat Transformer (AHT), a technology able to effectively recover and revalorize around $50 \%$ of low temperature waste heat, to obtain a higher temperature heat source, which can be reused in the industrial process. That way, the system works at three temperature levels: the high temperature level (revalorized heat, to be reused in the process), the medium temperature level (waste heat source temperature), and the low temperature level (heat sink temperature). This can be observed in Fig. 1, where the theoretical integration of the Indus3Es system in an industrial process is depicted.

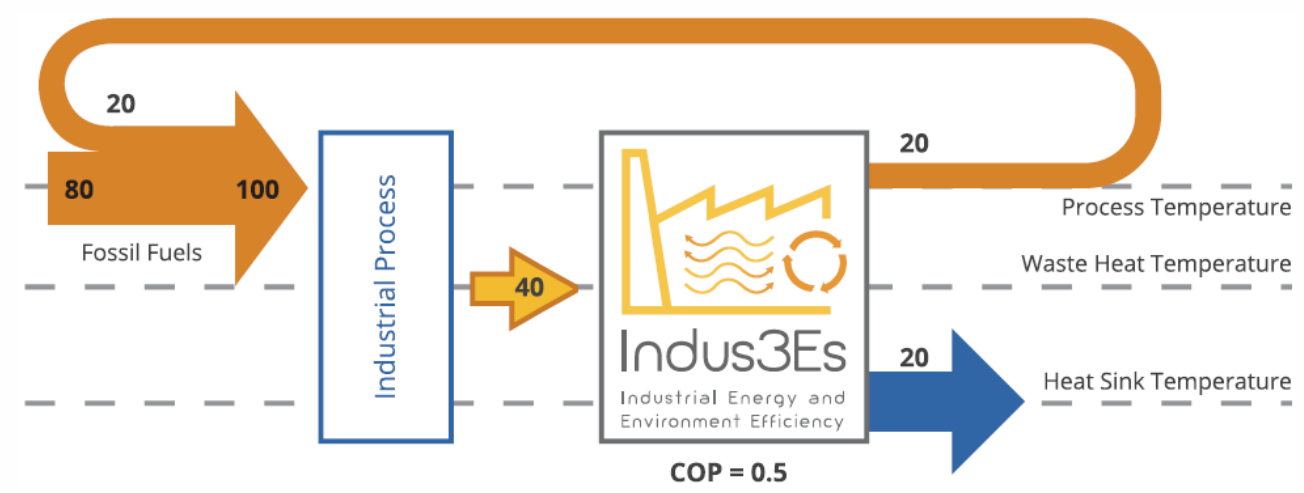

Fig. 1: Scheme of the Indus3Es project. 
As can be seen in Fig. 2, Absorption Heat Transformer system consisting of an evaporator, a condenser, a generator, an absorber and a solution heat exchanger operates in the reverse of Absorption Heat Pump. In the system, refrigerant vapour is produced in the evaporator heated by a low grade waste heat source. The refrigerant vapour is absorbed in the refrigerant-absorbent $(\mathrm{H} 2 \mathrm{O} / \mathrm{LiBr})$ solution that enters the absorber at a strong concentration and leaves it at weak concentration. The heat of absorption is transferred to the feed water of the absorber and boosts its temperature. The weak solution is transferred to the generator where some refrigerant vapour is removed, returning the solution to the strong state. Low grade heat is supplied to the generator to provide energy for desorption. The vaporized refrigerant is condensed in the condenser and pumped to higher pressure region where it is evaporated by the waste heat. The evaporated refrigerant is then absorbed in the absorber at a higher temperature, rising the temperature of the solution above the waste heat source temperature.

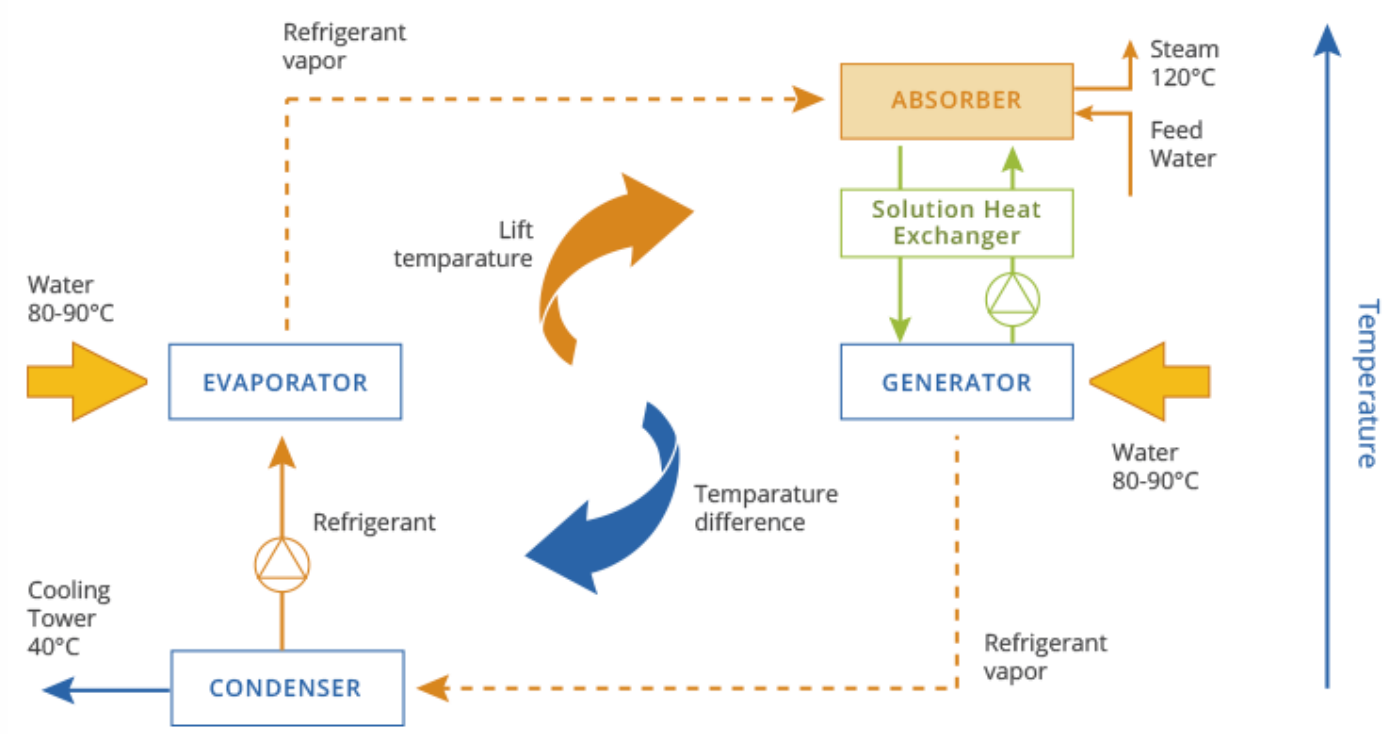

Fig. 2: Absorption Heat Transformer Cycle.

Indus3Es system will have a relevant impact making possible an energy efficiency increase and reduction of primary energy consumption of most energetic intensive industries in Europe.

The embodied energy, the environmental footprint of the products and the manufacturing costs of energy intensive industries will be reduced, increasing the competitiveness of European products. Moreover, it will allow a sustainable economic activity for local "auxiliary" companies, usually SMEs, in high added value services related to the energy efficiency measures for industry. Studies will be carried out through the project to maximize the future commercialization and exploitation of the developed technology and system. During the project the AHT technology will be demonstrated in a real environment, under working conditions of a Tüpras' refinery.

The consortium of the Indus3Es Project consists of 10 partners: 4 Research Organisations (Tecnalia, TU Berlin, Circe and Technion), 3 SMEs (Bs Nova, Aiguasol and PNO) and 3 large industries (Tupras, Repsol and Fertinagro). The Research organisations in charge of the main fundamental research activities related to the envisaged developments are highly experienced on absorption technology and heat transformers systems; the companies will bring the business spirit and industrial knowledge, while the large companies will be key to ensure a successful demonstration and replication of the project results.

PNO, as innovation and technology transfer organisation, will lead dissemination and communication activities and will support companies in exploitation issues, ensuring the project impact among the stakeholders and preparing a favorable framework for future exploitation of the project results, assuring alignment and smooth flows of information and providing close integration with Europe's research and innovation programmes, platforms and organisations. Tecnalia, one of the main Research \& Technology Organizations (RTO) in Europe, is the Coordinator of the project, and leads several technical activities in different stages of the project. 


\section{Results and discussion}

One of the first activities performed in the framework of the Indus3Es project was the analysis of applicability of the developed technology in different potential industrial processes, considering SPIRE (Sustainable Process Industry through Resource and Energy Efficiency) and non-SPIRE sectors. For this purpose, the different sectors were analyzed in depth, focusing principally on temperature levels, capacities and possible heat sources and heat sinks, which can suit AHT requirements. Results showed that many industrial processes are suitable for integration of AHT technology. Sectors which are especially promising are the petrochemical, pulp\&paper, and ceramics sector; followed by water and non-ferrous sectors, where it can be integrated in special applications. Cement and steel sectors showed too high temperatures for AHT integration.

Some processes of interest for integration of AHT in the petrochemical industries include their use in distillation columns or combination of AHT in heat exchanger networks, having a high potentiality to be used in these kinds of plants, due to the amount of hot and cold streams and the high capacity of the processes. Regarding the pulp\&paper industry, it is possible to recover heat from the steam leaving the cyclones in the pulping process, to be revalorized and used in the drying section. In the ceramics sector, heat recovery from the exhaust gases of the cooling process could be used to drive an AHT, for energy use in the drying chamber. AHT can be used in the water sector in certain processes, such as multieffect distillation and multi-stage flash distillation processes, or water desalination. Finally, in the non-ferrous sector, AHT integration could be more difficult, but the possible applications would be linked to cooling systems and external steam grids close to the waste heat generation.

The new developments for Heat Transformer Technology will be demonstrated in real conditions in the refinery of Tüpras in Turkey. For the purpose of defining the demonstration site, different processes within Tüpras refinery have been analysed, and the most suitable one has been chosen for the purpose, described as follows. Revalorized heat will be used to heat boiler feed water entering to the deaerator (or existing steam generator) from $\sim 65^{\circ} \mathrm{C}$ to higher than $110^{\circ} \mathrm{C}$ (preferably to $145^{\circ} \mathrm{C}$ ) to decrease the load of steam consumption in the deaerator (or boost the steam production in existing steam generator). The waste heat source temperature is around $100^{\circ} \mathrm{C}$. At the same time, in this case for dissipation source cooling water at approximately $25^{\circ} \mathrm{C}$ is considered. The scheme for integration of the AHT in the demonstration site can be seen in Fig.3. The nominal operating input temperatures for the AHT are $95^{\circ} \mathrm{C}$ for generator and evaporator, $130^{\circ} \mathrm{C}$ for absorber, and $25^{\circ} \mathrm{C}$ for condenser.

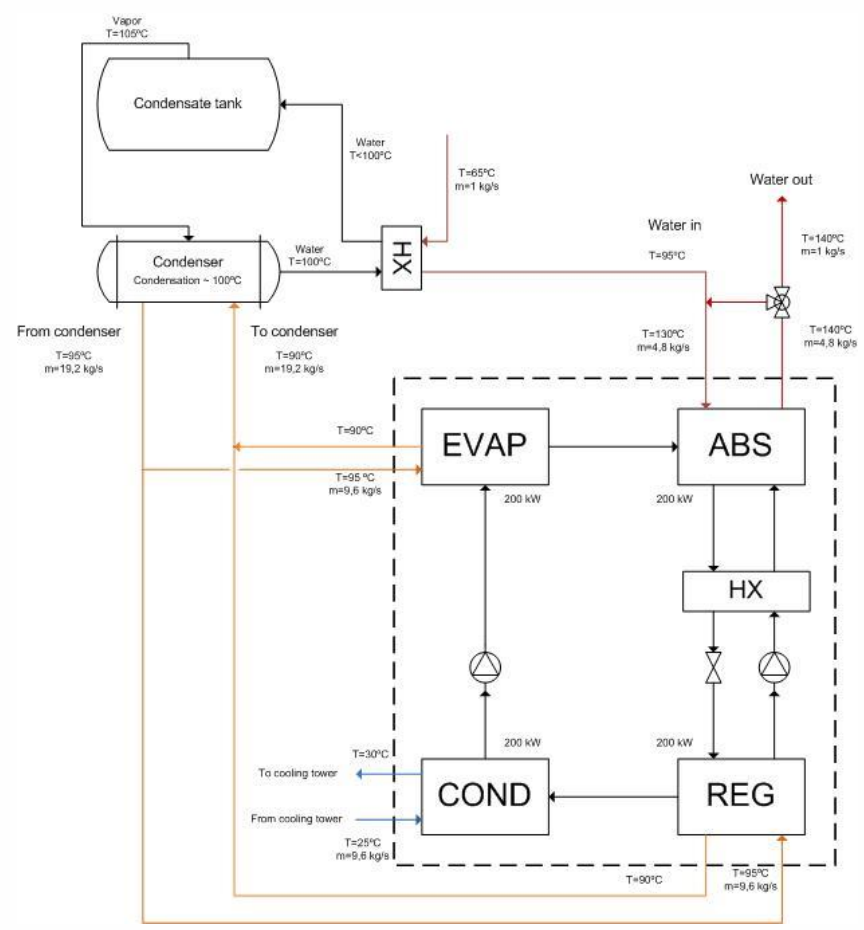

Fig. 3: Scheme of application of the AHT in the demonstrator. 
Experimental testing has been carried out in an existing Absorption Heat Converter prototype, which can work in Heat Transformer mode, in order to characterize its operation in nominal conditions (in the defined demonstration conditions), and also with variations of the external streams' flow rates and temperatures, and the solution flow rate. For the nominal conditions, COPs in the range between 0.42 and 0.47 can be expected.

A thermodynamic model of the whole AHT cycle has been developed and compared with the experimental results, adjusting the model with those, in order to make a sensitivity analysis showing the effect of the variation of some of the parameters when the complete AHT model is simulated around nominal conditions. Also, individual detailed models of each of the components of the AHT have been developed, being able to predict the operation of each one of them tube by tube. A comparison of these models with the experimental results has been done as well.

For the new prototype design, some innovations will be implemented in the AHT components. The generator will be a hybrid one, consisting on a pool boiling part (higher heat transfer coefficient) and a falling film part (more common in absorption technology). Also, enhanced surfaces will be used in the tubes, which will lead to more compact units. The condenser will use enhanced surfaces as well. The absorber and evaporator will use nozzles to atomize the solution and refrigerant respectively, enhancing the adiabatic absorption process in the case of the absorber. For the purpose of characterizing the new innovations, specially designed test benches have been built in Tecnalia (Low Pressure Vessel: Generator-Condenser) and Technion (High Pressure Vessel: Absorber-Evaporator), with low capacity of around $10 \mathrm{~kW}$.

This is the first stage of the design process. The testing of the vessels separately will allow for the characterization of each of the components in detail, to be able to size a higher capacity machine. Concretely, a $50 \mathrm{~kW}$ capacity AHT will be sized based on the measurements of the components. This medium size AHT will be tested in the laboratory of TU Berlin, and in the final stage the design of the machine for the demonstrator will be carried out, with a capacity of $200 \mathrm{~kW}$ revalorized heat.

Testing of the Low Pressure Vessel has been carried out in Tecnalia's laboratory, in nominal conditions and imposing variable external temperatures and flow rates to generator and condenser, and also variable solution flow rates. The falling film part of the generator has been tested for bare and enhanced tubes, showing an increase of around $30 \%$ capacity when using enhanced surfaces. The developed tests have been used for the sizing of the $50 \mathrm{~kW}$ Low Pressure Vessel. The testing of the High Pressure Vessel is under progress in Technion facilities. The experimental measurements will be, in turn, used for the sizing of the $50 \mathrm{~kW}$ High Pressure Vessel. These two vessels will be manufactured in the following months for being installed in the laboratory of TU Berlin, where the complete $50 \mathrm{~kW}$ capacity AHT will be tested. The testing of the $50 \mathrm{~kW}$ capacity AHT at laboratory level will be the intermediate step prior to the design and construction of the $200 \mathrm{~kW}$ AHT, to be installed in Tüpras' installations, and tested in real conditions for several months.

\section{Acknowledgements}

Indus3Es is a project funded by the European Commission. This project has received funding from the

European Union’s Horizon 2020 Research and Innovation program under Grant Agreement n ${ }^{\circ} 680738$.

\section{References}

[1] P. Donnellan, K. Cronin, E. Byrne, "Recycling waste heat energy using vapour absorption heat transformers: A review," Renewable and Sustainable Energy Reviews, vol. 42, pp. 1290-1304, 2015.

[2] I. Horuz, B. Kurt, "Absorption heat transformers and an industrial application," Renewable Energy, vol. 35, pp. 2175-2181, 2010.

[3] J. Yin, L. Shi, M. Zhu, L. Han, "Performance analysis of an absorption heat transformer with different working fluid combinations," Applied Energy, vol. 67, no. 3, pp. 281-292, 2000. 